\title{
Endoscopic Findings in Children with Isolated Lower Gastrointestinal Bleeding
}

\author{
Ari Silbermintz ${ }^{1 *}$, Manar Matar ${ }^{1 *}$, Amit Assa ${ }^{1,2}$, Noam Zevit $^{1,2}$, Yael Mozer Glassberg ${ }^{1,2}$ and Raanan Shamir ${ }^{1,2}$ \\ ${ }^{1}$ Institute of Gastroenterology, Nutrition and Liver Diseases, Schneider Children's Medical Center of Israel, Petach Tikvah, ${ }^{2}$ Sackler Faculty of \\ Medicine, Tel Aviv University, Tel Aviv, Israel
}

Background/Aims: Colorectal polyps are a common cause of lower gastrointestinal bleeding in children. Our aim was to study the causes of isolated lower gastrointestinal bleeding and to analyze the characteristics of the colorectal polyps found in our cohort. Methods: We retrospectively reviewed colonoscopic procedures performed between 2007 and 2015. Children with isolated lower gastrointestinal bleeding were included in the study.

Results: A total of 185 colonoscopies were performed for isolated lower gastrointestinal bleeding. The median patient age was 8 years, and 77 patients (41.6\%) were found to have colonic polyps. Normal colonoscopy findings were observed and acute colitis was detected in $77(41.6 \%)$ and $14(7.4 \%)$ patients, respectively. Single colonic polyps and 2-3 polyps were detected in $73(94.8 \%)$ and $4(5.2 \%)$ patients with polyps, respectively. Of the single polyps, 69 (94.5\%) were juvenile polyps, among which 65 (94.2\%) were located in the left colon. Conclusions: Single left-sided juvenile polyps were the most common cause of isolated lower gastrointestinal bleeding in our study. It was rare to find multiple polyps and polyps proximal to the splenic flexure in our cohort. A full colonoscopy is still recommended in all patients in order to properly diagnose the small but significant group of patients with pathologies found proximal to the splenic flexure. Clin Endosc 2019;52:258-261

Key Words: Colitis; Colonic polyps; Colonoscopy; Juvenile polyps; Rectal bleeding

\section{INTRODUCTION}

Lower gastrointestinal bleeding is a common reason for colonoscopy in children. ${ }^{1}$ The common etiologies of lower gastrointestinal bleeding in children include anal fissures, ulcerative colitis, and colonic polyps. Etiologies of painless isolated lower gastrointestinal bleeding with normal bowel movements may be secondary to colonic polyps and Meckel's diverticulum.

Received: February 11, 2018 Revised: October 23, 2018

Accepted: December 17, 2018

Correspondence: Ari Silbermintz

Institute of Gastroenterology, Nutrition and Liver Diseases, Schneider Children's Medical Center of Israel, 14 Kaplan Street, Petach Tikvah 49202, Israel

Tel: +972-3-925-3673, Fax: +972-3-925-3104, E-mail: asilbermintz@gmail.com

ORCID: https://orcid.org/0000-0002-7801-7983

*These authors contributed equally to this study.

cc This is an Open Access article distributed under the terms of the Creative Commons Attribution Non-Commercial License (http://creativecommons.org/ licenses/by-nc/3.0) which permits unrestricted non-commercial use, distribution, and reproduction in any medium, provided the original work is properly cited.
Colonic polyps in children are mainly solitary benign juvenile polyps requiring endoscopic removal to prevent possible sequelae. ${ }^{1}$ The peak age of diagnosis of juvenile polyps is between 2 and 5 years, with a male predominance. ${ }^{1,2}$ Juvenile polyps represent $70 \%-80 \%$ of colonic polyps found in children, and $60 \%-70 \%$ of them are solitary. ${ }^{3-5}$ Solitary juvenile polyps tend to be located in the rectosigmoid colon; however, $35 \%$ have been reported to be located in the proximal colon. ${ }^{6-9}$

Much of the information in the literature on colonoscopic findings in lower gastrointestinal bleeding involved children with a board range of complaints. Our aim was to assess the findings on colonoscopies performed for isolated lower gastrointestinal bleeding in otherwise healthy children.

\section{MATERIALS AND METHODS}

We retrospectively reviewed all colonoscopic procedures performed between October 2007 and October 2015 in pa- 
tients 0-18 years old at the Institute of Gastroenterology, Nutrition and Liver Diseases, Schneider Children's Medical Center of Israel.

Only patients with isolated lower gastrointestinal bleeding were included in our study. Children with characteristics of inflammatory bowel disease such as diarrhea, arthritis, aphthous stomatitis, perianal disease, weight loss, or increased serum inflammatory markers were excluded. In addition, patients with a known polyposis syndrome or other underlying diseases that may affect the bowel, such as graft versus host disease, were also excluded. Abdominal pain and constipation (without an apparent anal fissure) were considered nonspecific symptoms and were not excluded.

The recorded information included patient demographics, clinical presentation, laboratory test results, colonoscopy findings, histology, complications, and completeness of the endoscopic procedure. Colonoscopy was considered to be complete if it included an examination of the cecum or terminal ileum. Anemia was defined by age and sex. ${ }^{10}$

Statistical analysis of data was performed using the SPSS 24 software program. Frequency and proportion were used to describe categorical characteristics. Median values and interquartile range (IQR) were used to describe quantitative characteristics. Scale variables were analyzed using a $t$-test for parametric and the Mann-Whitney test for nonparametric distributed variables. Categorical outcomes were compared using the chi-square test or the Fisher exact test. A $p$-value of $<0.05$ was considered statistically significant. The study was approved by the Rabin Medical Center Institutional Review Board (no. 14-0268).

\section{RESULTS}

A total of 388 colonoscopies for lower gastrointestinal bleeding were evaluated. Of them, 203 colonoscopies were excluded from the study because of other complaints in addition to lower gastrointestinal bleeding. These complaints included a history of chronic disease (80) including inflammatory bowel disease and a post-organ transplant status; intermittent diarrhea presenting with hypoalbuminemia, anemia, and elevated inflammatory markers (83); intermittent diarrhea with hypoalbuminemia (20); perianal disease (2); arthritis (2); weight loss and anemia (8); constipation with anal fissures (7); and Blue rubber bleb nevus syndrome (1). A total of 185 colonoscopies met our criteria for isolated lower gastrointestinal bleeding during the study period. All of these were complete colonoscopies. The median patient age was 8 years (IQR, $4.9-12.5$ years), and 88 ( $47.6 \%)$ were female patients.

A total of 77 of 185 patients (41.6\%) with isolated lower gastrointestinal bleeding were found to have colonic polyps. Normal colonoscopy findings, acute (transient) colitis, and ulcerative colitis were observed in 77 (41.6\%), 14 (7.4\%), and $6(3.2 \%)$ patients, respectively. Other infrequent findings are summarized in Table 1.

Among the 77 patients found to have colonic polyps, single

Table 1. Infrequent Colonoscopic Findings in Our Cohort

\begin{tabular}{ll}
\hline Finding & Number (\%) \\
\hline Hemorrhoids & $2(1 \%)$ \\
Perianal fissures & $3(1.6 \%)$ \\
Reactive lymphoid hyperplasia & $1(1 \%)$ \\
Single erosion in the ileocecal valve & $1(0.5 \%)$ \\
Eosinophilic colitis & $1(0.5 \%)$ \\
Prolapse of the rectal mucosa & $1(0.5 \%)$ \\
Prominent vessel in the rectosigmoid & $1(0.5 \%)$ \\
Unspecified inflammatory stenosis in the left & $1(0.5 \%)$ \\
colon & \\
\hline
\end{tabular}

Total

colonoscopies

185

Fig. 1. Endoscopic and histologic findings in our cohort. 
colonic polyps were detected in $73(94.8 \%)$ and 2-3 polyps were found in 4 (5.2\%) patients (Fig. 1). Sixty-nine (94.5\%) of the single polyps were juvenile polyps (Fig. 1), of which 65 $(94.2 \%)$ were located in the left colon, $2(2.9 \%)$ in the transverse colon, and 2 (2.9\%) in the ascending colon (Fig. 2). One patient was noted to have a polyp in the left colon that could not be retrieved by the endoscopist for pathologic evaluation. Three patients had a single filiform lymphatic polyp that was located in the left colon. Of the 73 patients with a single polyp, 69 (95.5\%) had the polyp in the left colon. All 4 patients with multiple polyps were found to have juvenile polyps. In 3 of the 4 patients, the polyps were located in the left colon; in the fourth patient, 2 polyps were noted in the left colon and 1 was found in the cecum. Of the 77 patients with colonic polyps, 72 (93.5\%) had only left-sided colonic polyps.

Children with polyps had a median age of 5.5 years (IQR, 3.9-8.1 years), whereas those without polyps had a median age of 10.9 years (IQR, 5.8-15.7 years, $p=0.0001$; Table 2). Hemoglobin results from before the colonoscopy were available for 165 of the 185 children. Children with polyps were found to have a lower hemoglobin level (mean $12.1 \pm 1.4 \mathrm{mg} / \mathrm{dL}$ ) than those without polyps (mean $12.59 \pm 1.45 \mathrm{mg} / \mathrm{dL})(p=0.037)$. Anemia was detected in $58 \%$ of the children with polyps and in $42 \%$ of those without polyps. However, this difference was not statistically significant (Pearson chi-square test, $p=0.053$ ).

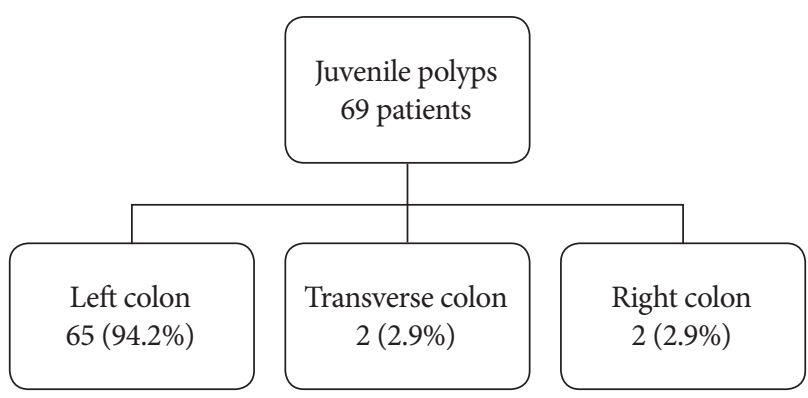

Fig. 2. Distribution of juvenile polyps in the colon.
The only complication noted in this cohort was a postpolypectomy bleeding event that was effectively treated with the application of a hemostatic clip in 1 patient.

\section{DISCUSSION}

Our study investigated children who underwent colonoscopy for isolated lower gastrointestinal bleeding with no diarrhea. The findings in our cohort provide additional data to the limited literature, as follows: (1) The most common abnormal finding was colorectal polyps (noted in $42 \%$ of the patients). (2) The vast majority of these were single juvenile polyps located in the left colon. (3) Only 4 children had multiple polyps, with no patient having more than 3 polyps. Of these, only 1 child had a polyp proximal to the splenic flexure. (4) Of the patients with a single polyp, only 4 children had a polyp proximal to the splenic flexure. Lastly, overall, only 5 of all 77 polyps (6.5\%) were located proximal to the splenic flexure.

The findings of our cohort stand in contrast to the study by Fox et al., who reported that $60.9 \%$ of juvenile polyps were single and $66.6 \%$ of these polyps were located in the left colon while the remaining $33.3 \%$ were located proximal to the splenic flexure. ${ }^{11} \mathrm{~A}$ less pronounced difference was noted when our results were compared with the study by Poddar et al., who demonstrated $76 \%$ solitary polyps with $85 \%$ of these being located in the rectosigmoid. ${ }^{12}$ These studies differ from ours in that they reviewed all colonoscopies that demonstrated polyps without regard to the clinical presentation and included patients with polyposis syndromes. A recent study reported that only $19.5 \%$ of patients with painless lower gastrointestinal bleeding had polyps, and that when patients with an additional complaint of abdominal pain with no change in bowel movements were included in the analysis, only $14.8 \%$ were found to have polyps. ${ }^{13}$ In contrast, our study included patients undergoing colonoscopy for isolated lower gastroin-

Table 2. Epidemiological, Demographic, and Clinical Characteristics of the Patients

\begin{tabular}{lccc}
\hline & Patients with polyps $(\boldsymbol{n}=\mathbf{7 7})$ & Patients without polyps $(\boldsymbol{n}=\mathbf{1 0 8})$ & $\boldsymbol{p}$-value \\
\hline Age: median (IQR) & 5.5 yr $(3.95-8.1$ yr $)$ & 10.9 yr $(5.8-15.7$ yr $)$ & 0.0001 \\
Female: $n$ (\%) & $41(43.2 \%)$ & $47(53.2 \%)$ & 0.192 \\
Hemoglobin: g/dL (SD) & $12.13 \pm 1.45$ & $12.6 \pm 1.45$ & 0.037 \\
Albumin: mg/dL (SD) & $4.2 \pm 0.92$ & $4.44 \pm 0.42$ & 0.073 \\
Constipation & $3(3.9 \%)$ & $9(8.3 \%)$ & 0.365 \\
Anemia $^{\text {a) }}$ & $40(58 \%)$ & $41(42.7 \%)$ & 0.053 \\
Hypoalbuminemia $^{\text {a) }}$ & $2(3.9 \%)$ & $2(2.4 \%)$ & 0.635 \\
\hline
\end{tabular}

IQR, interquartile range; SD, standard deviation.

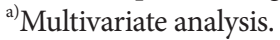


testinal bleeding, and we believe that excluding patients with polyposis syndromes and those with lower gastrointestinal bleeding associated with other complaints is likely a major reason for the different results obtained in our study.

Multiple studies have reported that juvenile polyps were more common in male patients. ${ }^{3,4,713}$ Our study, however, did not demonstrate a statistically significant difference between male and female patients.

Fox et al. reported neoplasia in 3.9\% of their cohort. ${ }^{11}$ All of these cases involved patients with more than 5 polyps. ${ }^{10}$ In our study, there were no cases of neoplasia and no patients with more than 3 polyps. This difference may be explained by the difference in the composition of the cohorts and the absence of patients with more than 3 polyps in our study. Overall, this indicates that when colonoscopy is performed for painless lower gastrointestinal bleeding, neoplasia is rarely, if at all, found.

Of interest, there were $6(3.2 \%)$ patients with ulcerative colitis in our study. Three of them had proctitis. This rate is lower than that reported in a recent study that analyzed patients with painless rectal bleeding with or without abdominal pain but with no change in bowel movements, in which inflammatory bowel disease was present in $10.7 \%$ of the patients. ${ }^{13}$

Currently, pancolonoscopy is the recommended method for the evaluation of recurrent painless rectal bleeding in children. ${ }^{14}$ All patients in this study had complete colonoscopy. As result, we found a small but significant minority (6.5\%) of patients with polyps proximal to the splenic flexure.

The main limitation of this study stems primarily from its retrospective design that did not allow complete data collection for several variables. Despite this limitation, our pediatric isolated lower gastrointestinal bleeding cohort study is one of the few studies that assessed the findings on colonoscopies performed for this indication.

In conclusion, we found that single left-sided juvenile polyps are the most common cause of isolated lower gastrointestinal bleeding in children. It was rare to find multiple polyps and polyps proximal to the splenic flexure in our cohort. It is an accepted practice to perform a full colonoscopy in patients presenting with isolated lower gastrointestinal bleeding. Despite the large majority of a left-sided pathology noted in our study, a full colonoscopy is still recommended in order to properly diagnose the small but significant group of patients with pathologies found proximal to the splenic flexure.

\section{Author Contributions}

Conceptualization: Ari Silbermintz, Manar Matar, Amit Assa, Noam Zevit, Yael Mozer Glassberg, Raanan Shamir

Data curation: AS, MM

Formal analysis: AS, MM

Investigation: AS, MM

Methodology: AS, MM, AA, NZ, YMG, RS

Project administration: AS, MM, RS

Resources: AS, MM

Supervision: AS, MM, RS

Validation: AS, MM, AA, NZ, YMG, RS

Visualization: AS, MM

Writing-original draft: AS, MM

Writing-review\&editing: AS, MM, AA, NZ, YMG, RS

\section{REFERENCES}

1. Torres P, Rossomando A, Delpretti F, et al. [The usefulness of endoscopic polypectomy of rectosigmoid and colon in childhood]. G E N 1991;45:51-54.

2. El-Shabrawi MH, El Din ZE, Isa M, et al. Colorectal polyps: a frequently-missed cause of rectal bleeding in Egyptian children. Ann Trop Paediatr 2011;31:213-218.

3. Lee HJ, Lee JH, Lee JS, Choe YH. Is colonoscopy necessary in children suspected of having colonic polyps? Gut Liver 2010;4:326-331.

4. Thakkar K, Fishman DS, Gilger MA. Colorectal polyps in childhood. Curr Opin Pediatr 2012;24:632-637.

5. Lin CH, Wu RS, Lin WC, Wu SF, Chen AC. Colonoscopic polypectomy of colorectal polyps in children under general anesthesia. Kaohsiung J Med Sci 2009;25:70-76

6. Mandhan P. Sigmoidoscopy in children with chronic lower gastrointestinal bleeding. J Paediatr Child Health 2004;40:365-368.

7. Sahn B, Bitton S. Lower gastrointestinal bleeding in children. Gastrointest Endosc Clin N Am 2016;26:75-98.

8. Haghi Ashtiani MT, Monajemzadeh M, Motamed F, et al. Colorectal polyps: a clinical, endoscopic and pathologic study in Iranian children. Med Princ Pract 2009;18:53-56.

9. Tam YH, Lee KH, Chan KW, Sihoe JD, Cheung ST, Mou JW. Colonoscopy in Hong Kong Chinese children. World J Gastroenterol 2010;16:1119-1122.

10. Brugnara C, Oski FA, Nathan DG. Disorders of erythrocyte production. In: Orkin SH, Nathan DG, Ginsburg D, Look AT, Fisher DE, Lux SE IV, eds. Nathan and Oski's hematology of infancy and childhood. 7th ed. Philadelphia (PA): WB Saunders; 2009. p. 455-520.

11. Fox VL, Perros S, Jiang H, Goldsmith JD. Juvenile polyps: recurrence in patients with multiple and solitary polyps. Clin Gastroenterol Hepatol 2010;8:795-799.

12. Poddar U, Thapa BR, Vaiphei K, Singh K. Colonic polyps: experience of 236 Indian children. Am J Gastroenterol 1998;93:619-622.

13. Campbell AM, Sugarman I. Does painless rectal bleeding equate to a colonic polyp? Arch Dis Child 2017;102:1049-1051.

14. Kleinman RE, Goulet OJ, Mieli-Vergani G, Sanderson IR, Sherman PM, Shneider BL. Walker's pediatric gastrointestinal disease. 6th ed. Raleigh (NC): People's Medical Publishing House USA; 2018.

\section{Conflicts of Interest}

The authors have no financial conflicts of interest. 